CARDIOVASCULAR MEDICINE

\title{
Circulating concentrations of cardiac proteins indicate the severity of congestive heart failure
}

\author{
T Goto, H Takase, T Toriyama, T Sugiura, K Sato, R Ueda, Y Dohi
}

Heart 2003;89:1303-1307

See end of article for authors' affiliations

Correspondence to: Dr Y Dohi, Internal Medicine and Molecular Science, Graduate School of Medical Sciences, Nagoya City University, Kawasumi, Mizuho-cho, Mizuho-ku, Nagoya 4678601, Japan; ydohi@ med.nagoya-cu.ac.jp

Accepted 5 June 2003
Objective: To test the hypothesis that myocardium specific proteins may be useful markers for evaluating the severity of congestive heart failure.

Methods: Serum concentrations of myosin light chain I (MLC-I), heart fatty acid binding protein (H-FABP), creatine kinase isoenzyme MB (CK-MB), and troponin $\mathrm{T}(\mathrm{TnT})$ and plasma concentrations of brain natriuretic peptide (BNP) were determined in 48 patients with acute deterioration of congestive heart failure, both before and after effective treatment.

Results: Before treatment, MLC-I (mean (SEM) 3.2 (2.2) $\mu \mathrm{g} / \mathrm{l}), \mathrm{H}-\mathrm{FABP}(9.0$ (3.5) $\mu \mathrm{g} / \mathrm{l}), \mathrm{TnT}(30$ (21) ng/ I), and BNP (761 (303) ng/l) were higher than the normal reference range, and concentrations of CK-MB $(5.4(2.9) \mu \mathrm{g} / \mathrm{l})$ were near normal. Treatment of congestive heart failure with conventional medication significantly decreased the concentrations of MLC-I (1.2 (0.3) $\mu \mathrm{g} / \mathrm{I}, \mathrm{p}<0.0001), \mathrm{H}-\mathrm{FABP}(6.0(2.0) \mu \mathrm{g} / \mathrm{l}$, $\mathrm{p}<0.0001), C K-M B(2.9$ (1.5) $\mu \mathrm{g} / \mathrm{l}, \mathrm{p}<0.0001)$, TnT (9 (1) ng/l, $\mathrm{p}<0.001)$, and BNP (156 (1 18) ng/l, $p<0.0001$ ). The decreases in H-FABP and CK-MB concentrations after treatment correlated with the decrease in BNP concentrations $(p<0.05)$. The absolute concentrations of MLC-I, H-FABP, CK$M B$, and TnT correlated positively with those of BNP $(p<0.01)$.

Conclusions: These findings suggest that MLC-I, H-FABP, CK-MB, and TnT may be used as reliable markers for the evaluation of the severity of congestive heart failure.
$\mathrm{T}$ he incidence of congestive heart failure (CHF) has increased greatly over the past two decades. ${ }^{1}$ Although significant advances have been made in the treatment of $\mathrm{CHF}^{2}$ the mortality and morbidity rates remain high, with one year mortality rates of $40 \%$ or higher in patients with advanced CHF. ${ }^{3}$

A number of clinical studies have shown that CHF results in neurohormonal alterations such as increased plasma concentrations of noradrenaline, atrial natriuretic peptide, and brain natriuretic peptide (BNP) or activation of the renin-angiotensin system. ${ }^{4}{ }^{5}$ Of these, BNP appears to be the most powerful neurohormonal predictor of left ventricular function and prognosis. ${ }^{6-8}$ However, myocardial structure is also altered in CHF. Non-contiguous areas of myocardial cell death and foci of replacement fibrosis are typical morphological changes in advanced CHF. ${ }^{10}$ Structural abnormalities in viable myocytes are also observed in non-ischaemic CHF, including hypertrophied myocardial cells, degeneration of subcellular organelles, lack of contractile materials, and increased cytoskeletal elements. ${ }^{9}$ These morphological changes may be accompanied by an increase in serum cardiac proteins. ${ }^{11-13}$ Myofibrillar proteins may be released into the circulation through degeneration of myofibrils, and cytosolic proteins may be released through leakage due to increased permeability of the membranes of injured myocytes. We were, thus, interested in a fundamental hypothesis that myocardium specific proteins may serve as useful markers in evaluating the severity of CHF.

The objective of the present study was to assess the clinical value of quantifying serum concentrations of the cardiac proteins myosin light chain I (MLC-I), heart fatty acid binding protein (H-FABP), creatine kinase isoenzyme $\mathrm{MB}$ $(\mathrm{CK}-\mathrm{MB})$, and troponin $\mathrm{T}(\mathrm{TnT})$ in the evaluation of the severity of CHF or the effect of medication on patients with CHF. We quantified the concentrations of these cardiac proteins in patients with acute deterioration associated with CHF both before and after appropriate treatment and compared the concentrations of the proteins with those of BNP.

\section{METHODS}

\section{Subjects and study design}

The study protocol was approved by the ethics committee of our hospital and each subject gave written informed consent before the start of the study. We studied 48 consecutive patients ( 27 men and 21 women with a mean (SEM) age of 69 (2) years) who were hospitalised with acute deterioration of CHF associated with dilated cardiomyopathy (table 1). All had impaired left ventricular systolic function (left ventricular ejection fraction $<50 \%$ on two dimensional echocardiography) and symptomatic heart failure (New York Heart Association (NYHA) class II to IV). The diagnosis of dilated cardiomyopathy was based on a carefully taken history, physical, laboratory, and radiological examinations, and findings on echocardiograms, coronary angiograms, and endomyocardial biopsy. No patients had stenosis greater than $75 \%$ on coronary angiograms. None of the patients exhibited clinical symptoms or signs suggestive of acute myocardial infarction, unstable angina, or acute myocarditis, and none had undergone cardiac or non-cardiac surgery in the three months before admission. Patients with severe pulmonary disease, acute or chronic renal or liver disease, uncontrolled hypertension, or inflammatory illness were excluded from the study. On admission, a clinical history, physical examination, ECG, echocardiogram, and laboratory data were obtained and a venous blood sample was taken for the measurement of MLC-I, H-FABP, CK-MB, TnT, and BNP

\footnotetext{
Abbreviations: $B N P$, brain natriuretic peptide; $C H F$, congestive heart failure; CK-MB, creatine kinase isoenzyme MB; H-FABP, heart fatty acid binding protein; MLC-I, myosin light chain I; NYHA, New York Heart Association; TnT, troponin T
} 
Table 1 Characteristics of patients

\begin{tabular}{|c|c|c|}
\hline & Acute phase & Stable phase \\
\hline NYHA class (I/II/III/IV) & $0 / 11 / 26 / 11$ & $32 / 15 / 1 / 0$ \\
\hline Body mass index $\left(\mathrm{kg} / \mathrm{m}^{2}\right)$ & $22.4(0.7)$ & $20.8(0.6)^{\star \star \star}$ \\
\hline Systolic blood pressure (mm Hg) & $140.0(3.8)$ & $118.5(3.0)^{* * *}$ \\
\hline Diastolic blood pressure (mm Hg) & $77.1(2.4)$ & $65.6(2.2)^{* *}$ \\
\hline Heart rate (beats/min) & $94.4(2.7)$ & $74.6(1.6)^{* * *}$ \\
\hline Cardiothoracic ratio (\%) & $63.8(0.9)$ & $56.6(1.2)^{* * *}$ \\
\hline Left atrial diameter $(\mathrm{mm})$ & $42.0(1.7)$ & $39.4(1.9)$ \\
\hline Diastolic left ventricular diameter $(\mathrm{mm})$ & $62.2(1.1)$ & $58.4(1.5)^{*}$ \\
\hline Systolic left ventricular diameter $(\mathrm{mm})$ & $53.0(1.3)$ & $46.1(1.7)^{\star \star \star}$ \\
\hline Ejection fraction (\%) & $29.4(1.8)$ & $42.7(2.4)^{* * *}$ \\
\hline Fractional shortening (\%) & $14.8(1.0)$ & $22.4(1.5)^{\star * *}$ \\
\hline
\end{tabular}

(acute phase). Then all patients received intensive treatment with intravenous administrations of diuretics, catecholamines, carperitide, or phosphodiesterase inhibitors. The evaluation was repeated after stabilisation of clinical findings and symptoms (stable phase). The time between the first and the second biochemical evaluation was 52 (5) days. Table 2 lists medications during the stable phase.

\section{Biochemical measurements}

For BNP measurement, $3 \mathrm{ml}$ of blood was transferred to a plastic tube containing $4.5 \mathrm{mg}$ of Na-EDTA and $1500 \mathrm{U}$ of aprotinin. For measurement of MLC-I, H-FABP, CK-MB, and TnT, $5 \mathrm{ml}$ of blood was transferred to plastic tubes. Serum and plasma samples were prepared within 30 minutes of blood sampling in a precooled centrifuge and were immediately frozen and stored at $-70^{\circ} \mathrm{C}$ until used for analysis. BNP and MLC-I concentrations were measured by radioimmunoassay (Shionoria BNP kit, Shionogi Co Ltd, Osaka, Japan and Myosin LI kit Yamasa, Yamasa Co, Chiba, Japan, respectively). H-FABP was measured by enzyme linked immunosorbent assay (ELISA) (Markit-M H-FABP, Dainippon Pharmaceutical Co, Chiba, Japan) and TnT was measured by electrochemiluminescence immunoassay (Elecsys Troponin T III, Roche Diagnostics GmbH, Mannheim, Germany). CK-MB was measured by immunochemiluminometric assay (Chemilumi ACS, Centaur, Bayer Medical Co Ltd, Tokyo, Japan). ${ }^{14}$ The analytical range, intraand interassay coefficients of variation, and normal reference range (99th centile of the control population) of the assay were: $4.0-2000 \mathrm{ng} / \mathrm{l}, 10.9 \%$ and $10.6 \%,<18.4 \mathrm{ng} / \mathrm{l}$ for BNP; $1.0-250 \mu \mathrm{g} / \mathrm{l}, \quad 3.0 \%$ and $5.1 \%,<2.5 \mu \mathrm{g} / \mathrm{l}$ for MLC-I; $1.1-250 \mu \mathrm{g} / \mathrm{l}, \quad 3.0 \%$ and $3.5 \%,<6.2 \mu \mathrm{g} / \mathrm{l}$ for H-FABP; $0.65-500 \mu \mathrm{g} / \mathrm{l}, 2.5 \%$ and $2.6 \%,<5.0 \mu \mathrm{g} / \mathrm{l}$ for CK-MB; and $10-25000 \mathrm{ng} / \mathrm{l}, 2.4 \%$ and $11.4 \%,<10 \mathrm{ng} / \mathrm{l}$ for TnT, respectively.

\section{Statistical analysis}

Data are expressed as mean (SEM). A paired Student's $t$ test was used to compare and analyse differences in continuous variables that had a normal distribution between the acute

\begin{tabular}{lr} 
Table 2 Medications & \\
\hline Angiotensin converting enzyme inhibitor & 19 \\
Angiotensin II type 1 receptor blocker & 15 \\
$\alpha$ Blocker & 5 \\
$\beta$ Blocker & 8 \\
Calcium channel blocker & 9 \\
Diuretics & 48 \\
Digitalis & 29 \\
Pimobendan & 3 \\
\hline
\end{tabular}

and stable phases. Because the distributions of MLC-I, HFABP, CK-MB, TnT, and BNP concentrations were skewed rightwards, median concentrations were computed for these parameters and expressed as median value (median absolute deviation). The significance of any difference in medians was assessed by the Wilcoxon signed rank test and correlations between these variables were assessed by the Spearman rank correlation test. A probability value of $p<0.05$ was considered significant.

\section{RESULTS}

On admission, all patients had worsening CHF with increased cardiothoracic ratios on chest radiographs and decreased left ventricular systolic function on echocardiograms (table 1). The circulating concentrations of BNP were higher than the normal reference range in all patients (fig 1). Biochemical analysis showed that serum concentrations of proteins derived from myocardial cells (MLC-I, H-FABP, and TnT) were higher than the normal reference range, and the concentrations of CK-MB were near normal (fig l).

All patients were successfully treated, resulting in improvement of their symptoms and physiological findings; the NYHA functional class, cardiothoracic ratio, and findings obtained from echocardiograms all improved (table 1). Effective medical treatment significantly decreased not only BNP but also MLC-I, H-FABP, CK-MB, and TnT (fig 1). The decrease in H-FABP and CK-MB, but not in MLC-I and TnT, concentrations after treatment significantly correlated with the decrease in BNP concentrations (fig 2). In addition, the absolute concentrations of MLC-I, H-FABP, CK-MB, and TnT correlated positively with those of BNP in patients with CHF (fig 3).

\section{DISCUSSION}

Structural changes associated with severe CHF are characterised by degeneration of subcellular organelles in viable myocytes and multiple foci of myocardial cell death. ${ }^{9}{ }^{10}$ Thus, we hypothesised that circulating concentrations of specific cardiac proteins may be useful as markers for the evaluation of the severity of CHF. The present study has shown that circulating concentrations of the cardiac proteins MLC-I, HFABP, CK-MB, and TnT correlated with the concentrations of BNP in patients with CHF. Since circulating BNP concentrations are increased in patients with CHF in proportion to the severity of the disease, the results of this study suggest that these specific cardiac proteins may be useful as powerful markers for determining the severity of CHF.

In the present study in patients with $\mathrm{CHF}$, the concentrations of MLC-I, H-FABP, CK-MB, and TnT were higher on admission (acute phase) than under the stable conditions achieved after appropriate treatment. The results are compatible with previous reports that circulating concentrations of 

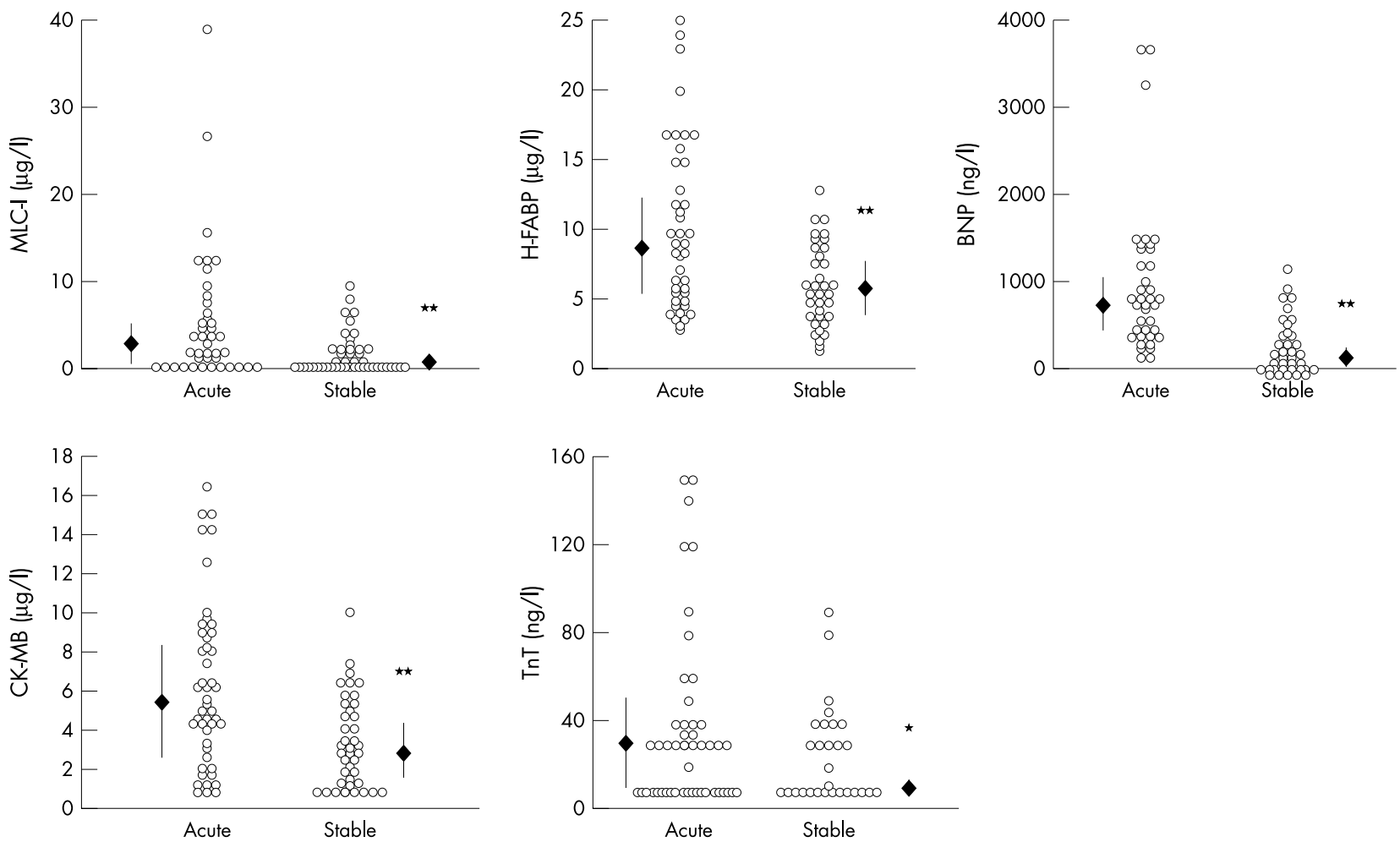

Figure 1 Serum concentrations of myosin light chain I (MLC-I), heart fatty acid binding protein (H-FABP), creatine kinase isoenzyme MB (CK-MB), troponin $\mathrm{T}(\mathrm{TnT})$, and brain natriuretic peptide (BNP) before (acute) and after effective treatment (stable) in patients with congestive heart failure. Open circles: scattergram showing data in each patient; closed diamonds and vertical bars: median value (median absolute deviation). ${ }^{*} p<0.001$, ${ }^{* *} \mathrm{p}<0.0001$ versus acute phase.
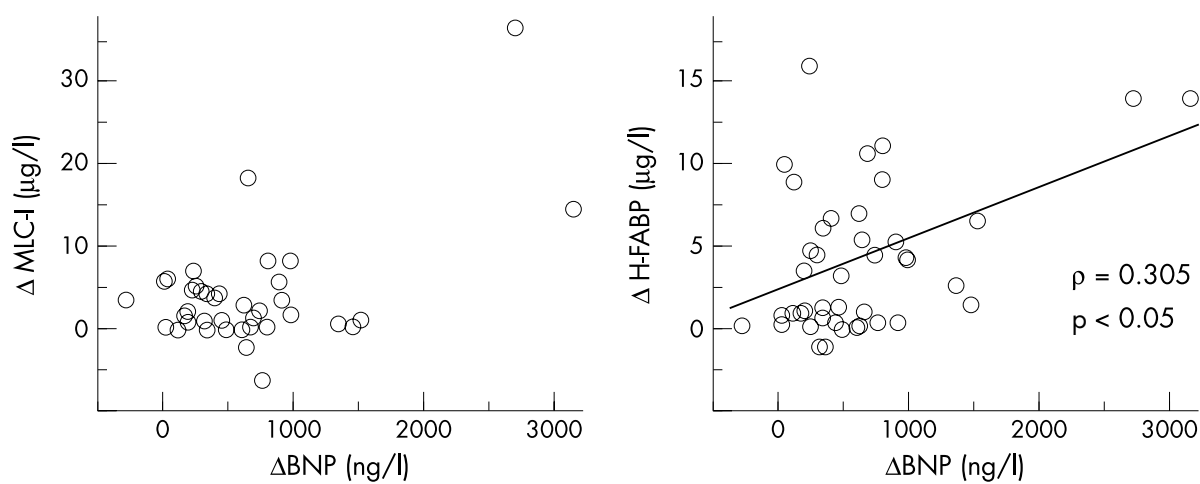

Figure 2 Correlations between the decrease in the concentrations of specific cardiac proteins and BNP after effective treatment of patients with congestive heart failure. The decrease in the concentrations of $\mathrm{H}-\mathrm{FABP}$ and CK-MB, but not of MLC-I and TnT, correlate with that in BNP.
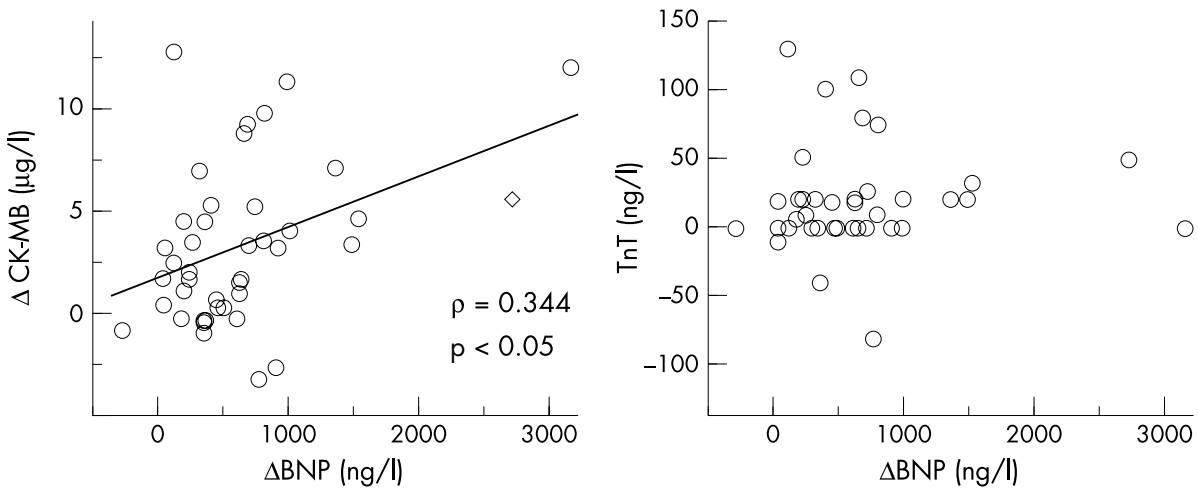

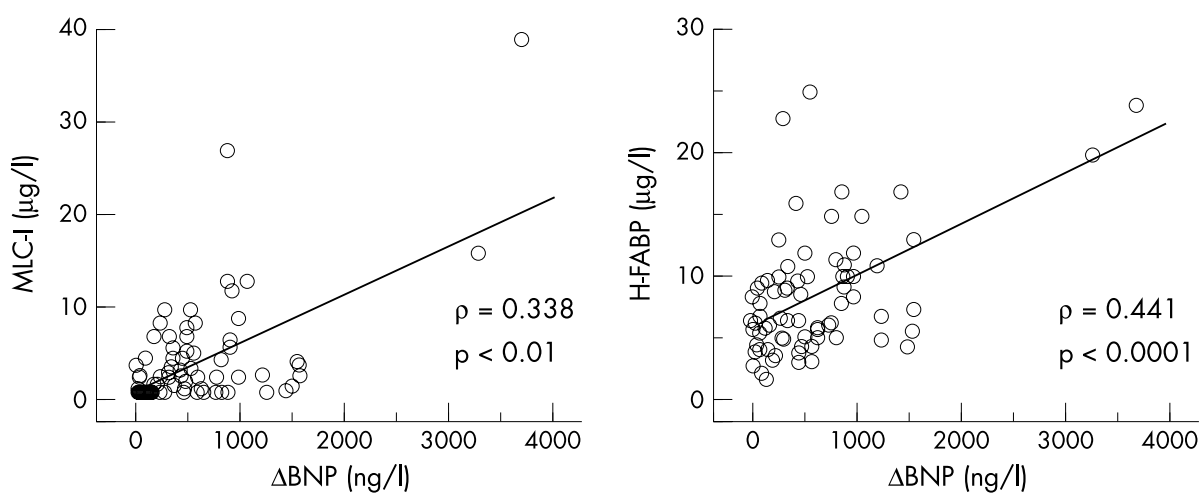

Figure 3 Correlations between the absolute values of specific cardiac proteins and of BNP in patients with congestive heart failure. The circulating concentrations of MLC-I, H-FABP, CK$M B$, and $\operatorname{TnT}$ correlate with those of BNP.
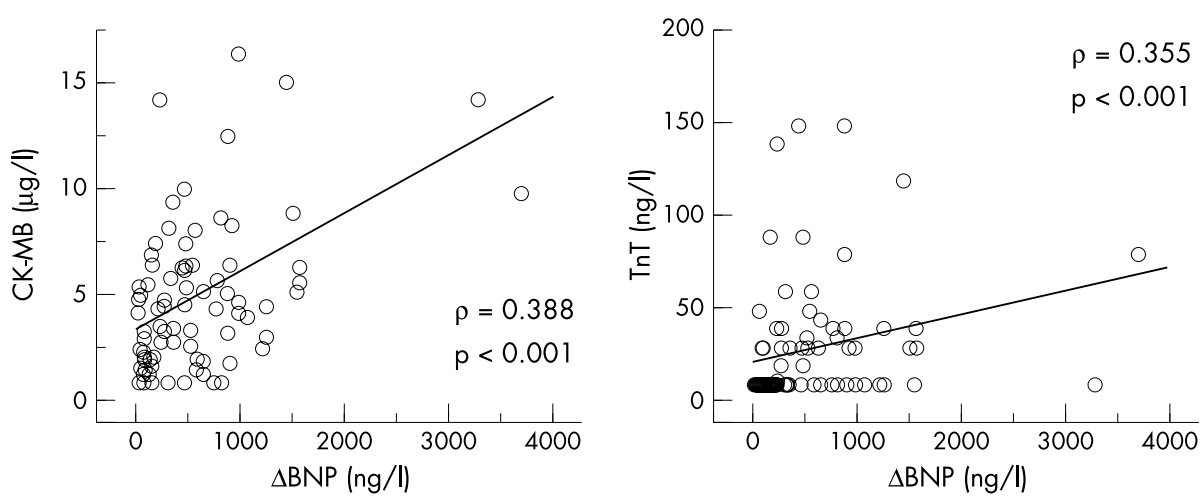

troponin I, TnT, and MLC-I are increased in advanced CHF. ${ }^{11-13}$ These proteins are specific to myocardial cells, suggesting that the increase in protein concentrations reflects cellular injury of the myocardium in advanced CHF. The presence of myocardial cytosolic proteins such as H-FABP and CK$\mathrm{MB}$ in serum from patients with severe CHF implies that permeability of the membrane of injured myocytes has been compromised and that the release of the cytosolic proteins into the circulation may be caused by leakage. ${ }^{15}$ However, this concept does not explain the appearance of MLC-I or TnT in the circulation, as the majority of these proteins are present in the contractile apparatus and not in cytosol. ${ }^{13}$ In severe CHF, myofibrils may degenerate and, subsequently, the myofibrillar components may be released into the circulation. ${ }^{11}{ }^{13}$ Indeed, degeneration of hypertrophied myocardial cells has been reported in human dilated cardiomyopathy. ${ }^{9}$ The mechanisms of ongoing myocardial damage in patients with CHF have not been established, but experimental models provide insight into the cellular and molecular mechanisms that contribute to the progression of CHF. Microcirculatory abnormalities of coronary arteries have been implicated in the aetiologies of human cardiomyopathy. ${ }^{16}$ These may be contributing factors to focal myocardial necrosis and replacement fibrosis. ${ }^{13}$ Recently, other mechanisms have been identified that also mediate the progressive impairment of cardiac structure and function including neurohormonal factors, oxidative stress, and some cytokines. ${ }^{17}$ Each of these factors can promote cardiac cell death by producing either myocyte necrosis ${ }^{18}{ }^{19}$ or myocyte apoptosis through activation of specific genetic pathways. $^{2021}$ These structural changes may accelerate left ventricular dysfunction, which increases left ventricular filling pressure resulting in secretion of BNP. Thus, it is hardly surprising that circulating concentrations of these specific cardiac proteins correlate with those of BNP in CHF.

Appropriate treatment greatly decreased the concentrations of MLC-I, H-FABP, CK-MB, and TnT, suggesting that the cardiac markers are quite sensitive to changes in left ventricular haemodynamic dysfunction. Strikingly, the decrease in H-FABP and CK-MB concentrations correlated with the decrease in BNP concentrations. This indicates that concentrations of the marker proteins appear to be related to left ventricular filling pressures and can give clinically important information for the treatment of CHF. Since BNP concentrations may serve as a useful prognostic indicator in patients with $\mathrm{CHF}^{7}{ }^{822}$ the marker proteins may also provide useful information on the prognosis of CHF. BNP and the marker proteins may have a complementary role in the prognostic assessment ${ }^{23}$ because $\mathrm{BNP}$ and the marker proteins provide intrinsically different information. However, further studies are necessary to confirm this hypothesis.

The interpretation of the present results is limited by the small number of patients studied. However, the clear correlation between the absolute concentrations of the specific cardiac protein markers and of BNP and between the decrease in the concentrations of the cardiac markers and of BNP shows that cardiac proteins may serve as reliable markers for the evaluation of the severity of CHF or the effect of treatment of CHF. Since BNP concentrations do not appear to indicate the severity of $\mathrm{CHF}$ in some cases, measurement of the circulating concentrations of these cardiac proteins in combination with BNP may provide more useful information than measurements of BNP concentrations alone in the management of patients with CHF.

\section{Authors' affiliations}

T Goto, H Takase, T Toriyama, T Sugiura, Department of Internal Medicine, Enshu General Hospital, Hamamatsu, Japan

K Sato, Department of Internal Medicine, Johoku Hospital, Nagoya, Japan

R Ueda, Y Dohi, Internal Medicine and Molecular Science, Graduate School of Medical Sciences, Nagoya City University, Nagoya, Japan 


\section{REFERENCES}

1 O'Connel JB, Bristow MR. Economic impact of heart failure in the United States: time for a different approach. J Heart Lung Transplant 1994;13:S107-12.

2 Cleland JG, Swedberg K, Poole-Wilson PA. Successes and failures of current treatment of heart failure. Lancet 1998:352(suppl 1):SI19-28.

3 Ho KK, Anderson KM, Kannel WB, et al. Survival after the onset of congestive heart failure in the Framingham heart study subjects. Circulation 1993;88: 107-15.

4 Francis GS Cohn JN, Johnson G, et al. Plasma norepinephrine, plasma rennin activity, and congestive heart failure: relations to survival and the effects of therapy in V-HeFT II. The V-HeFT VA cooperative studies group. Circulation 1993;87:VI40-8.

5 Schrier RW, Abraham WT. Mechanisms of disease: hormones and hemodynamics in heart failure. N Engl J Med 1999;341:577-85.

6 Wei CM, Heublein DM, Perrella MA, et al. Natriuretic peptide system in human heart failure. Circulation 1993;88:1004-9.

7 Tsutamoto T, Wada A, Maeda K, et al. Attenuation of compensation of endogenous cardiac natriuretic peptide system in chronic heart failure: prognostic role of plasma brain natriuretic peptide concentration in patients with chronic symptomatic left ventricular dysfunction. Circulation 1997;96:509-16

8 Burger MR, Burger AJ. BNP in decompensated heart failure: diagnostic, prognostic and therapeutic potential. Curr Opin Investig Drugs 2001:2:929-35.

9 Schaper J, Froede R, Hein St, et al. Impairment of the myocardial ultrastructure and changes of the cytoskeleton in dilated cardiomyopathy. Circulation 1991;83:504-14.

10 Beltrami CA, Finato N, Rocco $M$, et al. Structural basis of end-stage failure in ischemic cardiomyopathy in humans. Circulation 1994;89:151-63.

11 Missov E, Calzolari C, Pau B. Circulating cardiac troponin I in severe congestive heart failure. Circulation 1997;96:2953-8.
12 Setsuta K, Seino Y, Takahashi N, et al. Clinical significance of elevated levels of cardiac troponin $\mathrm{T}$ in patients with chronic heart failure. Am J Cardiol 1999;84:608-11

13 Del Carlo $\mathrm{CH}, \mathrm{O}^{\prime}$ Connor CM. Cardiac troponins in congestive heart failure. Am Heart J 1999; 138:646-53.

14 Piran U, Kohn DW, Uretsky LS, et al. Immunochemiluminometric assay of creatine kinase $M B$ with a monoclonal antibody to the $M B$ isoenzyme. Clin Chem 1987;33:1517-20.

15 Sohmiya K, Tanaka T, Tsuji R, et al. Plasma and urinary heart-type cytoplasmic fatty acid-binding protein in coronary occlusion and reperfusion induced myocardial injury model. J Mol Cell Cardiol 1993;25:1413-26.

16 Conway RS, Natelson BH, Chen WH, et al. Enhanced coronary vasoconstriction in the Syrian myopathic hamster supports the microvascular spasm hypothesis. Cardiovasc Res 1994;28:320-4.

17 Colucci WS. Molecular and cellular mechanisms of myocardial failure. Am J Cardiol 1997;80:15L-25L.

18 Ganote C, Armstrong S. Ischemia and the myocyte cytoskeleton: review and speculation. Cardiovasc Res 1995;27:1387-403.

19 Hein S, Sheffold T, Schaper J. Ischemia induces early changes to cytoskeletal and contractile proteins in diseased human myocardium. J Thorac Cardiovasc Surg 1995; 110:89-98.

20 Bing $\mathrm{OH}$. Hypothesis: apoptosis may be a mechanism for the transition to heart failure with chronic pressure overload. J Mol Cell Cardiol 1994;26:943-8.

21 Katz AM. Cell death in the failing heart: role of an unnatural growth response to overload. Clin Cardiol 1995; 18:36-44.

22 Koglin J, Pehlivanli S, Schwaiblmair M, et al. Role of brain natriuretic peptide in risk stratification of patients with congestive heart failure. J Am Coll Cardiol 2001;38:1934-41.

23 Ishii J, Nomura M, Nakamura Y, et al. Risk stratification using a combination of cardiac troponin $T$ and brain natriuretic peptide in patients hospitalized for worsening chronic heart failure. Am J Cardiol 2002;89:691-5

\section{IMAGES IN CARDIOLOGY}

\section{ICD lead implantation via persistent left superior vena cava}

A

67 year old man with chronic renal failure, hypertension and ischaemic heart disease was admitted for polymorphic ventricular tachycardia with syncope. Echocardiography revealed left ventricular hypertrophy with moderately impaired function. Amiodarone was administered for these arrhythmias, but recurrent episodes were experienced during haemodialysis. Therefore, an implantable cardioverter-defibrillator (ICD) was implanted at the left anterior chest on the other side of the shunt vein. Although the left cephalic vein was too small for insertion of a ventricular lead, an atrial lead was inserted from there, and the ventricular lead was inserted from the left subclavian vein. During the procedure, a persistent left superior vena cava (PLSVC) was detected incidentally, as both leads ran through an unusual left sided downward course. The Medtronic model $6945(65 \mathrm{~cm})$ screw-in ventricular lead was successfully affixed to the right ventricular apex by forming the stylet into a $U$ shape in the right atrium. The Intermedics model 438 $35 \mathrm{~S}(52 \mathrm{~cm})$ atrial lead was fixated in the right atrium (see panels). Atrial and ventricular sensing and stimulation thresholds were acceptable. Ventricular fibrillation induced by a $\mathrm{T}$ wave shock was successfully defibrillated twice with 20 joules of energy.
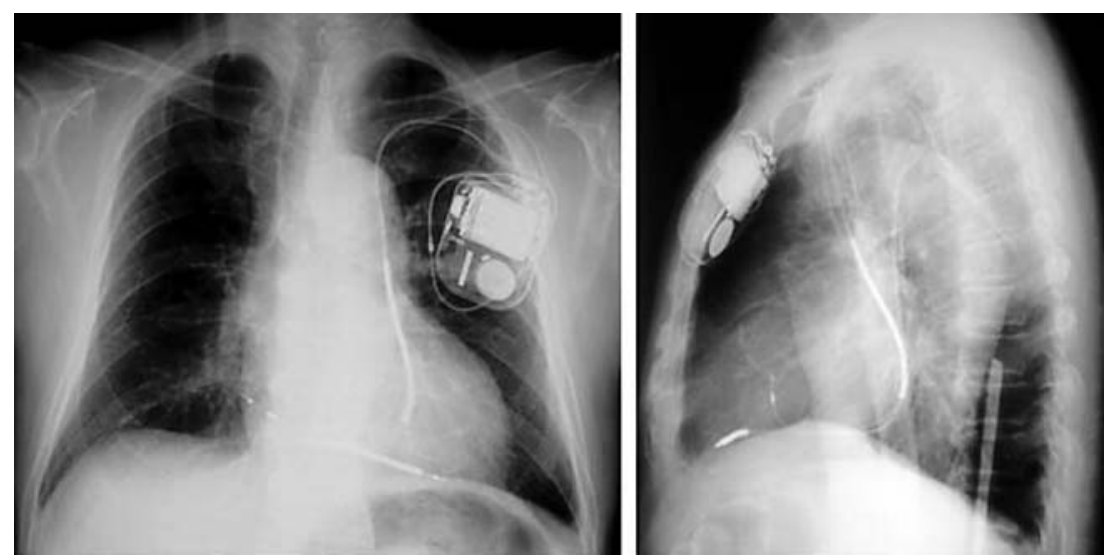

PLSVC is present in approximately $0.5 \%$ of the population, and a transvenous pacemaker or ICD implantation is sometimes difficult or even impossible in those cases. If transvenous implantation via PLSVC is intended, it is recommended to use a screw-in type electrode with sufficient length so that a loop in the right atrium can be formed for appropriate fixation to the right ventricle.

M Yasuda

Y Nakazato

H Daido

ynkzt@med.juntendo.ac.jp 